\title{
Diretrizes, estratégias de prevenção e rastreamento do câncer do colo do útero: as experiências do Brasil e do Chile
}

\author{
Cervical cancer guidelines, prevention and screening strategies: \\ experiences from Brazil and Chile
}

Itamar Bento Claro (https://orcid.org/0000-0002-6435-3302) ${ }^{1}$

Luciana Dias de Lima (https://orcid.org/0000-0002-0640-8387) ${ }^{2}$

Patty Fidelis de Almeida (https://orcid.org/0000-0003-1676-3574) ${ }^{3}$

${ }^{1}$ Instituto Nacional de Câncer José Alencar Gomes da Silva. Rua Marquês de Pombal $1257^{\circ}$ andar, Centro. 20230-240 Rio de Janeiro RJ Brasil. iclaro@inca.gov.br ${ }^{2}$ Departamento de Administração e

Planejamento em Saúde, Escola Nacional de Saúde Pública Sergio Arouca, Fundação Oswaldo Cruz. Rio de Janeiro RJ Brasil.

${ }^{3}$ Instituto de Saúde Coletiva, da Universidade Federal Fluminense. Niterói RJ Brasil.

\begin{abstract}
This article analyzes cervical cancer control policies and actions in Brazil and Chile, focusing on prevention and screening. We adopted a comparative approach to identify similarities and differences in guidelines and cervical cancer prevention and screening strategies between the two countries. We used the following data collection techniques: analysis of official documents and secondary data, consultations with experts, government officials and program coordinators, and literature review. The findings show that Chile has a well-structured program with centralized decision -making and a system that permits monitoring of actions. Brazil on the other hand faces ongoing issues with lack of coordination and shortcomin$g s$ in the follow-up of women with abnormal test results. The following challenges to consolidating cervical cancer screening stand out in Brazil: lack of active tracking of the target population; absence of a test quality assurance system; and inadequate follow-up of women with abnormal test results. Both countries need to increase coverage and implement organized screening.
\end{abstract}

Key words Cervical cancer, Screening, Public policy, Health systems
Resumo Este artigo analisa as políticas e ações de controle do câncer do colo do útero no Brasil e no Chile, com foco na prevenção e no rastreamento. Adotou-se a abordagem comparativa, buscando identificar semelhanças e diferenças entre as diretrizes e as estratégias de prevenção e rastreamento do câncer do colo do útero entre os países. A pesquisa compreendeu revisão bibliográfica, análise documental e de dados secundários e consultas a especialistas, técnicos e dirigentes do programa. Em que pesem as diferenças nas estratégias de rastreamento do câncer do colo do útero, o Chile possui um programa bem estruturado, com concentração de decisões no nivel nacional e um sistema que permite o monitoramento das ações. $O$ Brasil enfrenta recorrentes problemas relacionados à falta de uma coordenação das ações e falhas no segmento das mulheres com exames alterados. As principais dificuldades para a consolidação de programas de rastreamento do câncer do colo do útero (não realização da busca ativa da população em risco, ausência de sistema de controle de qualidade dos exames e seguimento inadequado de mulheres com resultados alterados) são mais evidentes no Brasil. Em ambos os países há necessidade de aumento da cobertura e implantação do rastreamento organizado.

Palavras-chave Neoplasias do colo do útero, Programas de rastreamento, Política pública, Sistemas de Saúde 


\section{Introdução}

O câncer do colo do útero (CCU) é um problema de saúde pública global com aproximadamente 570 mil casos e 311 mil mortes no mundo ${ }^{1}$. Cerca de $87 \%$ a $90 \%$ das mortes causadas pela doença ocorrem em países de baixa e média renda, expressando o componente de iniquidade social associado a esse tipo de câncer². Entretanto, as experiências internacionais mostram que é possível reduzir em até $80 \%$ o número de óbitos pela doença ${ }^{3}$.

Com esse propósito, a Organização Pan-Americana de Saúde (OPAS) propõe a implantação de programas nacionais de controle do CCU que abrangem ações e serviços de prevenção primária, deteç̧ão precoce, tratamento e cuidados paliativos ${ }^{4}$. Tais programas vêm sendo implantados na maioria dos países da América Latina desde os anos 1980, sem a obtenção dos resultados alcançados pelos países de alta renda ${ }^{3,5}$. Fatores relacionados ao acesso e à qualidade dos serviços de saúde, bem como ao nível socioeconômico das mulheres são apontados como responsáveis pelas dificuldades no controle do câncer na região ${ }^{6}$.

Este artigo tem como objetivo analisar os programas de controle do CCU no Brasil e no Chile, com foco nas diretrizes nacionais, estratégias de prevenção e rastreamento. $\mathrm{O}$ rastreamento do CCU está voltado para a diminuição da mortalidade e da incidência da doença, por meio da realização sistemática de testes em populações assintomáticas que visam a identificação, a confirmação e o tratamento de lesões precursoras ${ }^{7,8}$. Países que implantaram programas de rastreamento organizados reduziram a incidência para menos de dez casos por cem mil mulheres/ano'. Em cenários sem esta intervenção, as taxas de incidência podem ultrapassar 70 casos por cem mil mulheres ${ }^{1,10}$. Ressalta-se que as intervenções relativas à prevenção e ao rastreamento são fortemente condicionadas pelas desigualdades sociais $^{11}$ e demandam sistemas de saúde bem estruturados e organizados para garantir o acesso adequado à rede de serviços de saúde 5 .

Chile e Brasil implantaram programas nacionais para o controle do CCU em momentos distintos, respectivamente, a partir de 1987 e de 1998. A despeito dos dois países terem programas com propostas semelhantes, estas se desenvolveram em contextos específicos, condicionados por diferentes configurações dos sistemas de saúde ${ }^{12,13}$. É neste cenário que as diretrizes e as estratégias de prevenção e rastreamento do CCU são analisadas, procurando-se problematizar os desafios e resultados das ações implementadas.
Além da magnitude do CCU no contexto latino-americano, o estudo justifica-se pela necessidade de promover reflexão crítica para a qualificação de ações e a construção de estratégias voltadas para o diagnóstico e tratamento das lesões precursoras em tempo oportuno, que possam ter impacto na redução de mortes evitáveis, sobretudo entre mulheres com piores condições de vida.

\section{Método}

A pesquisa se caracteriza como um estudo de casos múltiplos, de natureza exploratória, centrado nas experiências do Brasil e do Chile. Adotou-se o método comparado para análise das diretrizes e estratégias de prevenção e rastreamento do $\mathrm{CCU}^{14,15}$, buscando-se identificar semelhanças e diferenças entre os países quanto às ações desenvolvidas. Além disso, por meio da comparação, pretendeu-se discutir as implicações de características contextuais e da configuração dos sistemas de saúde ${ }^{16}$ na ocorrência de problemas comuns e específicos, e nos resultados das ações implantadas em cada país.

Os dois casos foram selecionados por apresentarem as menores taxas de incidência e mortalidade por CCU na América do Sul, pioneirismo na organização de programas nacionais para o controle do CCU, exame citopatológico (Papanicolaou) como método utilizado para o rastreamento, diretrizes clínicas e organização das ações estabelecidas nacionalmente e representação no Grupo Operativo de Câncer do Colo do Útero na Rede de Institutos Nacionais de Câncer da América Latina (RINC).

As técnicas de pesquisa compreenderam análise de documentos e dados secundários, consultas a especialistas e revisão bibliográfica. Os documentos oficiais (atos normativos) sobre os programas nacionais de controle do CCU foram obtidos por meio da busca nos sites oficiais dos governos. Foram selecionados indicadores socioeconômicos e de saúde disponíveis no International Agency for Research on Cancer (IARC) (https://gco.iarc.fr/), na Comissão Econômica para a América Latina (CEPAL) (https://www. cepal.org/pt-br), na Organização para a Cooperação e Desenvolvimento Económico (OCDE) (http://www.oecd.org/), na Organização Mundial da Saúde (OMS) (https://www.who.int/es), no Banco Mundial (https://www.worldbank. org/) e nos sistemas de informação em saúde do Brasil (http://datasus1.saude.gov.br/) e do Chile 
(https://www.minsal.cl/). Em ambos os países, as consultas com especialistas, técnicos do Ministério da Saúde responsáveis pela coordenação das ações no nível central e profissionais responsáveis pelo programa no nível regional e local, foram realizadas no ano de 2019.

A revisão da literatura foi realizada nas bases de dados MEDLINE ${ }^{\circledR}$ (Medical Literature Analysis and Retrieval Sistema Online), LILACS ${ }^{\circledR}(\mathrm{Li}-$ teratura Latino-americana e do Caribe em Ciências da Saúde), Scopus ${ }^{\circledR}$ e no Catálogo de Teses e Dissertações da Coordenação de Aperfeiçoamento de Pessoal de Nível Superior (CAPES).

Este estudo foi aprovado pelo Comitê de Ética e Pesquisa da Escola Nacional de Saúde Pública Sergio Arouca, da Fundação Oswaldo Cruz.

\section{Resultados}

\section{Diretrizes nacionais do programa de controle do câncer do colo do útero}

Brasil e Chile possuem diretrizes nacionais estabelecidas para o controle do CCU, com definição de faixa etária da população-alvo, periodicidade de realização do exame e conduta para tratamento e seguimento das mulheres com exames alterados. As diretrizes têm o objetivo de reduzir a incidência, a morbidade, a mortalidade e melhorar a qualidade de vida de mulheres com CCU, além de fornecerem base científica sólida e atualizada para as equipes de saúde em aspectos relacionados à prevenção, diagnóstico precoce e tratamento da doença ${ }^{12,13}$.

As primeiras estratégias organizadas, no campo da política pública, de prevenção do CCU no Brasil, datam de 1984, no escopo de ações do Programa de Atenção Integral à Saúde da Mulher (Paism), estimulando a coleta de material para o exame citopatológico como procedimento de rotina na consulta ginecológica ${ }^{13}$. Em 1986, o Ministério da Saúde iniciou o projeto Expansão da Prevenção e Controle do Câncer Cervicouterino que possibilitou a ampliação da rede de coleta de material e da capacidade instalada de laboratórios de citopatologia em todo o país, estabelecendo a periodicidade e a faixa etária para o exame de prevenção do CCU $^{13}$. Em 1998, após avaliação do projeto de expansão, o MS instituiu o Programa Nacional de Combate ao Câncer de Colo do Útero (PNCCCU), coordenado pelo Instituto Nacional de Câncer (INCA), que ficou conhecido pelo nome da campanha denominada Programa Viva Mulher ${ }^{17}$. A denominação "Viva
Mulher" deixou gradativamente de ser utilizada, passando-se a utilizar o nome Programa Nacional de Controle do Câncer do Colo do Útero e de Mama e, posteriormente, Ações de Controle do Câncer do Colo do Útero e de $\mathrm{Mama}^{18}$, a serem desenvolvidas de forma descentralizada na Atenção Básica, principalmente a partir da expansão e fortalecimento Estratégia Saúde da Família.

No Chile, os esforços para a detecção precoce do câncer de colo do útero começaram em 1966, por meio de um acordo com a Faculdade de Medicina da Universidade do Chile, com o apoio da OPAS. Esse acordo possibilitou a expansão progressiva de laboratórios de citologia e a formação de recursos humanos nos três níveis de atenção. Após a avaliação dos resultados em 1987, as medidas implementadas foram reformuladas, com base nas recomendações da OPAS, dando origem ao Programa Nacional Pesquisa y Control del Cáncer. A periodicidade do exame Papanicolaou passou a ser trienal e a população-alvo mulheres entre 25 e 64 anos. As estratégias foram implementadas em um projeto piloto na Região Metropolitana, entre 1988 e 1994, e, após a avaliação, foram expandidas para todo o país ${ }^{19}$.

No Quadro 1, há o registro histórico dos principais marcos das políticas e ações de controle do câncer do colo do útero no Brasil e no Chile.

\section{Estratégias de prevenção, diagnóstico e tratamento das lesões precursoras}

Brasil e Chile contam com a vacinação contra o papilomavírus humano (HPV) como estratégia de prevenção. Em ambos os países, a vacina é gratuita e foi introduzida no calendário nacional de vacinação em 2014 e, desde 2019, está disponível para meninas e meninos. No Chile, a vacinação é obrigatória e realizada nas escolas, seguindo as recomendações internacionais para adolescentes, enquanto no Brasil é feita nas unidades básicas de saúde. Em 2019, o Chile alcançou uma cobertura de $79,6 \%$ para meninas na segunda dose e o Brasil, 47,4\%. O desconhecimento da população sobre a vacina, a falta de prescrição médica e as notícias equivocadas veiculadas sobre a sua segurança contribuem para a recusa da vacina por parte da população nos dois países ${ }^{20,21}$.

No Brasil, desde 1998, o método utilizado para o rastreio do CCU é a citologia convencional (exame de Papanicolaou) a cada três anos ${ }^{13}$. No Chile, desde 2015, existem duas orientações para os grupos populacionais específicos: a primeira é realizar o rastreamento com exame $\mathrm{Pa}$ panicolaou em mulheres na faixa etária entre 25 
a 64 anos, a cada três anos; e a segunda, para as áreas onde o teste de biologia molecular (DNA HPV) estiver disponível, realizá-lo em mulheres de 30 a 64 anos, a cada cinco anos ${ }^{12}$. Ao contrário de países onde há um programa organizado com recrutamento ativo da população-alvo, o rastre-

Quadro 1. Principais marcos do controle do câncer do colo do útero no Brasil e no Chile.

\begin{tabular}{|c|c|}
\hline Brasil & Chile \\
\hline 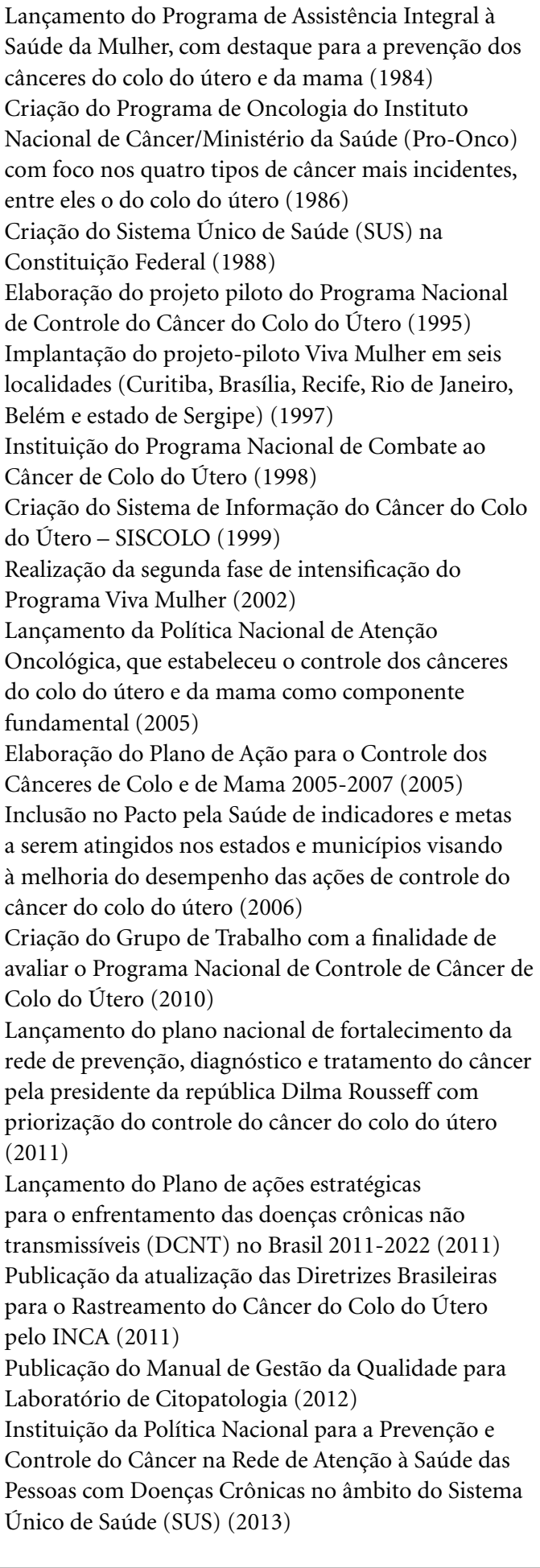 & 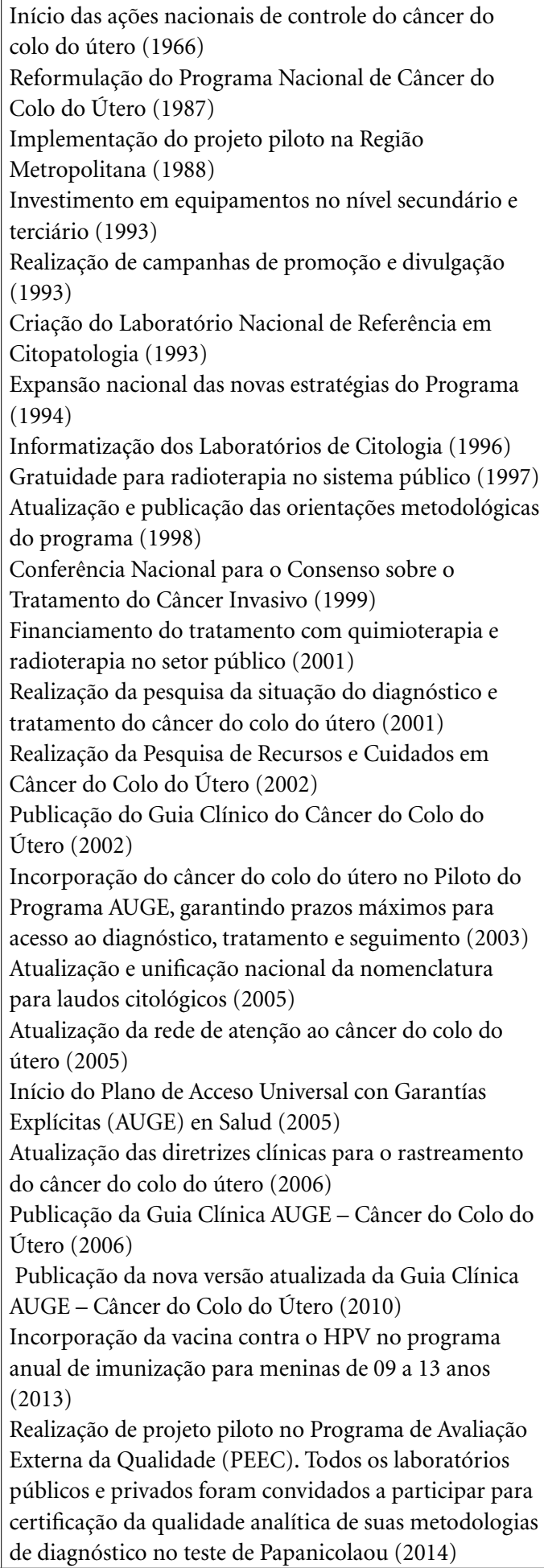 \\
\hline
\end{tabular}


Quadro 1. Principais marcos do controle do câncer do colo do útero no Brasil e no Chile.

\begin{tabular}{|l|l|}
\hline \multicolumn{1}{|c|}{ Brasil } & \multicolumn{1}{c|}{ Chile } \\
\hline Lançamento do Sistema de Informação de Câncer & Ampliação da vacina conta HPV para meninas até 13 \\
(SISCAN), uma versão em plataforma web que integra & anos (2015) \\
os Sistemas de Informação do Câncer do Colo do & Publicação da Guia Clínica AUGE - Câncer do Colo \\
Útero (SISCOLO) e do Câncer de Mama (SISMAMA) & do Útero (2015) \\
(2013) & Autorização do uso de teste DNA HPV para mulheres \\
Publicação da Portaria da QualiCito para definição & com mais de 30 anos (onde o teste estiver disponível) \\
de padrões de qualidade e avaliação da qualidade do & (2015) \\
exame citopatológico do colo do útero no âmbito do & Estudo piloto na Região de Valdívia para \\
SUS (2013) & implementação do rastreamento com teste de DNA \\
Publicação da Portaria que instituiu o Serviço de & HPV (2016) \\
Referência para Diagnóstico e Tratamento de Lesões & Publicação do Plano Nacional de Câncer 2018-2028 \\
Precursoras do Câncer do Colo do Útero (SRC) (2014) & (2018) \\
Início da campanha de vacinação contra o vírus HPV & Introdução do rastreio com DNA HPV em 15 serviços \\
em meninas entre 11 e 13 anos no Programa Nacional & de saúde no país, previsão de 2020 ser implantado no \\
de Imunizações (PNI) (2014) & Chile (2020) \\
Publicação da 2a edição revista, ampliada e atualizada & \\
das Diretrizes Brasileiras para o Rastreamento do & \\
Câncer do Colo do Útero (2016) & \\
Publicação da 2a edição revista e ampliada do & \\
Manual de Gestão da Qualidade para Laboratório de & \\
Citopatologia (2016) & \\
\hline
\end{tabular}

Fonte: Elaboração própria com base nos documentos consultados.

amento em ambos os países ocorre, de modo geral, de forma oportunista, a partir da procura ocasional e espontânea dos serviços de saúde por razões diversas que não o rastreamento do CCU.

No Brasil, desde 2001, a participação em um sistema de controle de qualidade externo consta como exigência para a contratualização de laboratórios prestadores de serviços para o SUS. Entretanto, em 2019, apenas dez de um total de 27 estados apresentaram informações sobre a realização do monitoramento externo da qualidade (MEQ) no Sistema de Informações Ambulatoriais do SUS (SIA/SUS) (http://datasus1.saude. gov.br/), que é o sistema de registro obrigatório para pagamento. No Chile, o MEQ é coordenado pelo Instituto de Salud Pública de Chile (ISP) e disponibiliza um programa de avaliação externa da qualidade para todos os laboratórios públicos e privados do país desde 1993, o que permite manter a qualidade e a confiabilidade do resultado dos exames no sistema público.

No Brasil, o sistema de informação de câncer existente desde 2013, SISCAN, é um sistema nominal, que permite o monitoramento e seguimento das mulheres que realizaram o exame citopatológico. Além disso, permite registrar, arquivar e sistematizar as informações referentes aos exames de rastreamento e também selecionar as amostras para monitoramento externo, favorecendo a criação de um programa de gestão da qualidade. Contudo, não está plenamente implementado no conjunto dos municípios e estados brasileiros. No Chile, há dois sistemas de informação utilizados para o monitoramento do programa: o Cito-Expert, que registra dados dos exames realizados em plataforma online, onde todos os profissionais autorizados podem verificar se a usuária realizou o rastreio conforme periodicidade estabelecida nas diretrizes nacionais; e o SIGGES, projetado para monitorar o cumprimento das metas estabelecidas pelo Ministério da Saúde.

No Brasil, os problemas relacionados às informações sobre o seguimento das mulheres com exames alterados são persistentes. Em 2010, apenas $23 \%$ das mulheres diagnosticadas, no ano anterior, tiveram o seguimento registrado no sistema de informação. No Chile, o cadastro obrigatório da população nos centros de atenção primária, para atendimento no sistema público de saúde, e o estabelecimento de prazos para realização de exames e tratamentos complementares, favorecem e determinam a realização do seguimento. $\mathrm{O}$ Chile ainda possui uma gestão altamente centralizada, o que facilita a coorde- 
nação das ações, ao contrário do Brasil, onde a gestão do programa é descentralizada com ações a cargo dos municípios e dos estados.

As características gerais das estratégias de prevenção e rastreamento do câncer do colo do útero no Brasil e no Chile estão sintetizadas no Quadro 2.

\section{Discussão}

Brasil e Chile são países marcados por grandes desigualdades socioeconômicas, apresentando ações e políticas de bem-estar social mais restritas se comparadas à média de países de renda média e alta o que se reflete, por exemplo, em importantes indicadores de educação, segurança, emprego, moradia e acesso aos serviços de saúde ${ }^{22,23}$. Tais desigualdades sociais impactam diretamente na magnitude do CCU e favorecem sua manutenção como importante problema de saúde pública em ambos os países ${ }^{24,25}$, refletindo-se na disparidade da ocorrência, no perfil epidemiológico, na sobrevida e na qualidade de vida após o diagnóstico ${ }^{26}$. O Quadro 3 apresenta alguns indicadores sociais e de financiamento em saúde selecionados para uma caracterização dos dois países.

No Brasil, o acesso às ações e serviços públicos de saúde são garantidos por um sistema público universal (Sistema Único de Saúde, SUS) que oferece cobertura para toda a população, sem exclusão de grupos populacionais. No Chile, para ter acesso ao sistema público de saúde (Fondo Nacional de Salud, FONASA), os trabalhadores e pensionistas devem contribuir com $7 \%$ dos seus rendimentos tributáveis ${ }^{27}$. As estratégias de garantia de acesso oportuno no Chile estão restritas às garantias de saúde do Plano AUGE, para tratamento das patologias listadas como prioridades no país. Para os demais serviços, há outro fluxo assistencial, em geral, com maiores tempos de espera. Ainda assim, o Plano AUGE, instituído pela reforma do sistema de saúde chileno em 2005, representou uma ampliação do acesso e estabeleceu um monitoramento ativo de metas ${ }^{27,28}$.

Em ambos os países, as desigualdades regionais configuram barreiras de acesso para parte da população. No Brasil, a população que reside em áreas rurais remotas, especialmente nas regiões Norte e Nordeste do país, enfrenta maior dificuldade na utilização de serviços de saúde, principalmente na atenção especializada ${ }^{29}$. No Chile, além das barreiras regionais de acesso e a centralização da assistência nas regiões metropolitanas, há impedimentos relacionados à contribuição financeira obrigatória e aos gastos com medicamentos, consultas médicas e exames que necessitam de copagamento ${ }^{30}$. Em ambos os países, as regiões distantes dos grandes centros metropolitanos apresentam as maiores taxas de incidência e mortalidade.

Embora tenham alcançado aumento da cobertura de exames para o diagnóstico precoce e do acesso ao tratamento das lesões precursoras

Quadro 2. Estratégias de prevenção e rastreamento do câncer do colo do útero no Brasil e no Chile, 2020.

\begin{tabular}{|c|c|c|}
\hline Componentes & Brasil & Chile \\
\hline Diretrizes clínicas & $\begin{array}{l}\text { Diretrizes Brasileiras para o } \\
\text { Rastreamento do Câncer do Colo do } \\
\text { Útero, } 2016 .\end{array}$ & Guía Clínica Cáncer Cervicouterino, 2015 \\
\hline População-alvo & Mulheres de 25 a 64 anos & Mulheres de 25 a 64 anos \\
\hline Método e Intervalo & $\begin{array}{l}\text { Citologia convencional (Papanicolaou) } \\
\text { a cada três anos, após dois exames } \\
\text { anuais negativos }\end{array}$ & $\begin{array}{l}\text { Papanicolaou em mulheres entre } 25 \text { a } 64 \text { anos, a } \\
\text { cada três anos. Triagem primária em mulheres } \\
\text { de } 30 \text { a } 64 \text { anos, com detecção de HPV e triagem } \\
\text { com Papanicolaou ou genotipagem de HPV 16- } \\
\text { 18, em intervalos de cinco anos (se disponível); }\end{array}$ \\
\hline Vacina contra HPV & $\begin{array}{l}\text { Incorporada ao calendário de } \\
\text { vacinação nacional do Sistema único } \\
\text { de Saúde em 2014. Atualmente } \\
\text { destinada a meninas e meninos de } 9 \text { a } \\
14 \text { anos de idade. } \\
\end{array}$ & $\begin{array}{l}\text { Incorporada desde 2014, é distribuída de forma } \\
\text { gratuita pelo Ministério da Saúde a todas as } \\
\text { meninas de } 11 \text { e } 12 \text { anos, no quarto e quinto } \\
\text { ano básico escolar. Atualmente destinada a } \\
\text { meninas e meninos. }\end{array}$ \\
\hline Meta de Cobertura & $80 \%$ da população-alvo & $80 \%$ da população-alvo \\
\hline $\begin{array}{l}\text { Sistema de } \\
\text { Informação }\end{array}$ & $\begin{array}{l}\text { Sistema de Informação do Câncer } \\
\text { (SISCAN) }\end{array}$ & $\begin{array}{l}\text { Sistema de Informação para a Gestão de } \\
\text { Garantias Explícitas de Saúde (SIGGES) }\end{array}$ \\
\hline
\end{tabular}

Fonte: Elaboração própria com base na bibliografia pesquisada. 
do CCU, o mesmo não ocorreu no que se refere à redução das taxas de incidência e mortalidade em comparação aos países de alta renda ${ }^{1}$. Em 2018 , com uma taxa estimada de 12,2 novos casos a cada cem mil mulheres em ambos os países, o CCU ocupava a quarta posição dos cânceres mais incidentes na população feminina no Brasil e a sexta posição no Chile ${ }^{1}$. O Gráfico 1 , com as taxas de mortalidade ajustadas pela população mundial, permite perceber que, apesar de os países apresen- tarem valores muito semelhantes desde o ano de 2010, partiram de patamares muito diferentes. $\mathrm{O}$ Chile, em 1980, apresentava taxa de mortalidade por CCU de 14,85 por cem mil mulheres, quase três vezes maior do que o Brasil com taxa de 5,20 por cem mil mulheres. A redução de mais de 50\% na mortalidade por CCU no Chile é decorrente das mudanças significativas nas políticas públicas de prevenção e tratamento implementadas no país. Outros aspectos importantes a considerar,

Quadro 3. Indicadores socioeconômicos e de financiamento em saúde em anos selecionados. Brasil e Chile, 2016 a 2020.

\begin{tabular}{|c|c|c|c|c|}
\hline \multicolumn{2}{|r|}{ Indicadores } & Ano & Brasil & Chile \\
\hline \multirow[t]{9}{*}{ Socioeconômicos } & População (em milhões) ${ }^{\mathrm{a}}$ & 2020 & 212.559 & 19.116 \\
\hline & População Feminina (em milhões) ${ }^{\mathrm{a}}$ & 2020 & 108.124 & 9.691 \\
\hline & 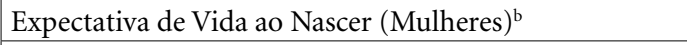 & 2016 & 77.80 & 83.10 \\
\hline & População alfabetizada de adultos ${ }^{c}$ & 2017 & $93.08 \%$ & $96.40 \%$ \\
\hline & Prevalência de tabagismo em mulheres ${ }^{\mathrm{c}}$ & 2016 & $10.1 \%$ & $34.2 \%$ \\
\hline & Paridade (nascimento por mulher) ${ }^{\mathrm{c}}$ & 2017 & 1.73 & 1.67 \\
\hline & Produto Nacional Bruto per capita ${ }^{c}$ & 2018 & 15.820 & 24.190 \\
\hline & Cobertura populacional (Sistema Público) \% & 2019 & 100.00 & $76.01^{\mathrm{b}}$ \\
\hline & Cobertura populacional (Sistema Privado) \% & 2019 & 24.20 & $17.97^{\mathrm{e}}$ \\
\hline \multirow[t]{5}{*}{ Financiamento } & Gasto total em saúde em \% PIB ${ }^{\mathrm{d}}$ & 2017 & 9.47 & 8.98 \\
\hline & Gasto público em saúde em $\%$ do PIB $^{d}$ & 2017 & 3.96 & 4.50 \\
\hline & Gasto público em saúde em \% do gasto total em saúded & 2017 & 42.00 & 50.00 \\
\hline & Gasto público em saúde per capita em US\$ & 2017 & 795.66 & 1.190 .55 \\
\hline & Desembolso direto em \% do gasto total em saúde ${ }^{\mathrm{d}}$ & 2017 & 27.46 & 33.55 \\
\hline
\end{tabular}

Fontes: a. United Nations DESA/PopulationDivision. World Population Prospects 2017. Disponível em: https://esa.un.org/ unpd/wpp/Download/Standard/Population/. Acesso em 12/11/2019; b. OECD. Stat. Disponível em: http://stats.oecd.org. Acesso em 12/11/2019; c.The World Bank. GNI per capita, PPP (current international \$). Disponível em: https://data.worldbank. org/indicator/NY.GNP.PCAP.PP.CD?name_desc=false. Acesso em 12/11/2019; d.WHO. Global Health Expenditure. Database. Disponível em: http://apps.who.int/nha/database/ViewData/Indicators/es. Acesso em 03/03/2020; e. Chile. Superintendencia de Salud. http://www.supersalud.gob.cl/664/w3-propertyvalue-6059.html

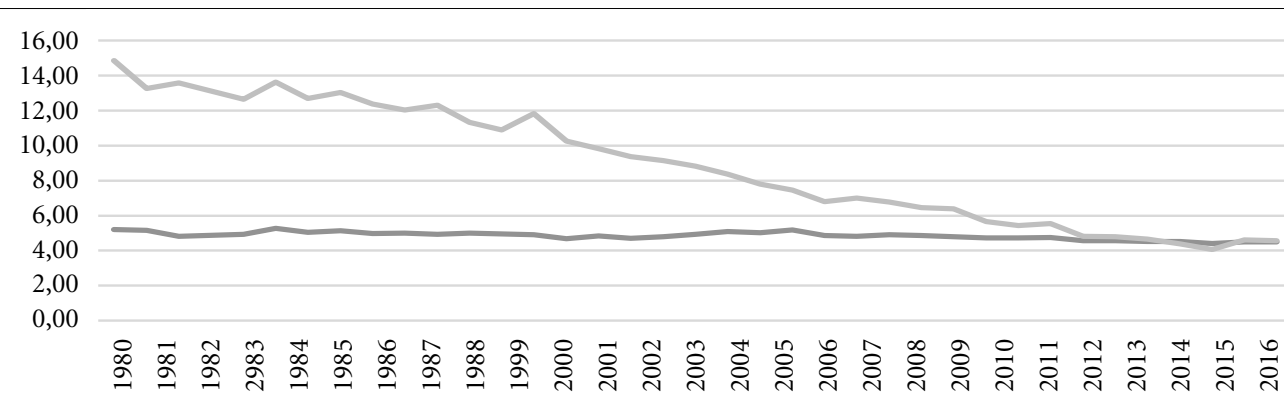

$\longrightarrow$ Brasil Chile

Gráfico 1. Evolução da taxa de mortalidade por câncer do colo do útero ajustada por idade pela população mundial. Brasil e Chile, 1980-2016.

Fonte: Ferlay J et al. (2020). Global Cancer Observatory: Cancer Today. Lyon, France: International Agency for Research on Cancer. Available from: https://gco.iarc.fr/today, accessed [03 May 2020]. 
além do impacto das políticas públicas de saúde, é a diminuição da proporção da população abaixo da linha de pobreza $(38,4 \%$, em 1990 , para $14,4 \%$, em 2011) e o aumento do Produto Interno Bruto (PIB) nas últimas décadas no Chile ${ }^{31}$.

Ambos os países estabeleceram como meta nacional a cobertura de $80 \%$ da população-alvo, considerada como a cobertura necessária para um real impacto na redução da incidência e da mortalidade. No Chile, a cobertura é calculada com base no sistema de informação nominal e tem se mantido próxima de $70 \%$. No Brasil, o percentual estimado de $80 \%$ tem como base as informações autorrelatadas em pesquisas nacionais, pois o país ainda não possui um sistema de informação nominal plenamente implantado. Os dois programas apresentam dificuldades para atingir a meta de cobertura e alcançar as mulheres da população-alvo que nunca realizaram o exame ou que o fizeram fora da periodicidade recomendada. Um programa de rastreamento de base populacional organizado que possibilite entrar em contato com as mulheres por meio de carta ou mensagens telefônicas ainda é uma agenda a ser cumprida em ambos os países.

O sistema de informação nominal é condição sine qua non para a realização do rastreamento organizado $^{32,33}$. No Brasil, a efetiva implantação do SISCAN é imprescindível para estruturação de um programa organizado que possibilite a realização do seguimento das mulheres com exames alterados e avaliação dos indicadores de cobertura. O fato de o Chile contar com um sistema de informação nominal plenamente implantado torna possível a realização do seguimento e essa é uma marcante diferença entre os dois países. Também o fato de haver metas estabelecidas e um forte controle ministerial sobre cada procedimento das garantias GES, torna obrigatório que todas as mulheres com exames alterados sejam alvo de busca ativa por parte dos serviços, para que se cumpram os prazos estabelecidos e os tratamentos efetuados de forma adequada. No Brasil, o caráter oportunístico do rastreamento, somado a problemas na qualidade dos registros nos sistemas de informações e à baixa adesão aos protocolos nacionais, acarreta dificuldades adicionais para o monitoramento, a avaliação e a organização das ações de controle do $\mathrm{CCU}^{34}$. A inconformidades entre os resultados dos exames e as condutas clínicas estabelecidas nas diretrizes nacionais, bem como importantes falhas em decorrência da desarticulação dos serviços assistenciais geram interrupções e atrasos no tratamento no contexto brasileiro ${ }^{35}$.
A garantia da qualidade do exame citopatológico é outro ponto fundamental no rastreamento do $\mathrm{CCU}^{36,37}$. Os estudos mostram que os indicadores de qualidade da maioria dos laboratórios no Brasil ainda apresentam parâmetros abaixo dos recomendados pelo Ministério da Saúde ${ }^{37}$ - No Chile, o monitoramento realizado parece refletir no índice de positividade, um parâmetro de análise que expressa a sensibilidade em detectar lesões na população rastreada. Para o ano de 2018, esse índice no Chile foi de 3,62\%, enquanto no Brasil foi de 2,76\%, abaixo do parâmetro nacional estabelecido como igual ou maior a $3 \%$ ${ }^{37}$. No Chile, a coordenação centralizada do MEQ no ISP favorece a organização e a qualidade do planejamento de um conjunto integrado de atividades em associação com a Universidade do Chile, cujo laboratório é acreditado pelo College of American Pathologists (CAP), um dos mais exigentes sistemas de avaliação internacional.

A Estratégia Saúde da Família apresenta-se como o eixo estruturante da Atenção Primária à Saúde e do sistema de saúde no Brasil, tendo papel fundamental na organização das ações para prevenção do CCU por meio de ações de educação em saúde, vacinação e rastreamento. ${ }^{13}$ Entretanto, um estudo censitário das unidades básicas de saúde (UBS) revelou que somente metade apresentava estrutura apropriada para a realização do exame preventivo e que apenas 30\% das equipes poderiam ser classificadas com prática adequada ao rastreamento, indicando insuficiência de equipamentos e insumos que restringem o escopo de ações do programa, sinalizando barreiras de acesso e qualidade à realização do rastreamento $^{38}$. A desarticulação entre os níveis de atenção é outro problema constantemente mencionado nas análises do sistema de saúde brasileiro e acarreta falhas no seguimento das mulheres ${ }^{39}$. Já o Chile se destaca pela maior institucionalização de instrumentos de coordenação do cuidado e, sobretudo, pela definição de protocolos elaborados pelo Ministério da Saúde ${ }^{40}$. E apesar de o Chile ter um dos sistemas de saúde mais desiguais da região ${ }^{41,42}$, a situação do CCU e das demais patologias consideradas prioritárias no Plano AUGE são exceções no contexto chileno.

No histórico do Programa, desde a sua criação até o momento presente, há uma dinâmica instável de gestão, que ora atribui papel coordenador ao INCA e ora a outra área técnica da própria estrutura do MS em Brasília. Essa alternância e a pouca definição sobre os papeis da coordenação em nível central compromete o acompanhamento, o monitoramento e a avaliação das ações. Si- 
tuação agravada pela dificuldade de estruturar e manter equipes gerenciais para o programa seja nas Secretarias Estaduais de Saúde (SES) seja nas Secretarias Municipais de Saúde (SMS). A gestão tem representado um desafio permanente à sustentabilidade das ações, refletindo-se no processo de planejamento e pactuação, na política de qualificação de recursos humanos e na garantia da continuidade do cuidado (p.9) ${ }^{28}$. Por fim, é possível constatar que a ausência de uma coordenação e acompanhamento amplo e sistemático das ações e de seus resultados, nos diversos níveis de gestão, tem sido um problema permanente no contexto brasileiro.

$\mathrm{Na}$ comparação entre os países, verifica-se que, no Brasil, alguns dos fatores identificados como principais responsáveis pelas falhas nos programas de rastreamento do CCU - não realização da busca ativa da população em risco, ausência de sistema de controle de qualidade dos exames e seguimento inadequado de mulheres com resultados alterados ${ }^{43}$ - são mais evidentes. A parcial implementação do SISCAN entre os municípios brasileiros que impossibilita o monitoramento dos exames realizados na população -alvo; a ausência de um sistema de controle de qualidade nacionalmente implantado e as falhas no seguimento das mulheres com exames alterados, agravam a situação no Brasil.

O Quadro 4 sistematiza algumas das principais características das políticas públicas de saúde e das estratégias e diretrizes para o controle do CCU no Brasil e no Chile.

\section{Considerações finais}

Ao se analisar as ações de detecção precoce do CCU no Brasil e no Chile, uma das principais lições aprendidas decorre da importância da garantia da qualidade do exame citopatológico e do seguimento da mulher com prazos estabelecidos para a confirmação diagnóstica e tratamento. A organização do programa, com coordenação bem definida e mecanismos de avaliação e monitoramento consolidados nacionalmente, também merecem destaque.

O programa chileno tem como característica uma gestão centralizada, continuidade do corpo técnico, padronização de protocolos, metas e indicadores de desempenho monitorados continuamente à nível nacional. Em termos de coordenação e sistematização de condutas, o programa do Chile se destaca em comparação ao brasileiro. No Brasil, falta uma linha clara de coordenação para monitoramento e avaliação de resultados como ocorre no Chile, com definição de responsabilidades entre os entes federados, sobretudo as gestões estaduais, o que fragiliza a configuração de um programa de ações nacionais.

Nos dois países, as maiores taxas de incidência e mortalidade são observadas entre os grupos mais vulneráveis, confirmando que o maior risco de CCU deve-se à indisponibilidade ou à limitação de acesso às ações de saúde. Embora os programas de controle de câncer possam reduzir as barreiras advindas das desigualdades sociais, não as eliminam e, por isso, é importante haver estratégias destinadas a aumentar a cobertura com intervenções voltadas aos grupos mais vulneráveis. Nesse aspecto, tanto o Brasil quanto o Chile necessitam de ações mais ousadas para o alcance das mulheres da população-alvo que permanecem à margem do rastreamento.

Em países com programa de rastreamento organizado e onde as ações e serviços de saúde estão disponíveis de forma universal para toda a população há menor impacto das desigualdades socioeconômicas nos resultados das estratégias de rastreamento ${ }^{44,45}$. O Brasil, apesar de contar com um sistema universal de saúde, no qual as ações de detecção precoce devem ser realizadas, sobretudo, pelas equipes da Estratégia Saúde da Família, capilarizadas e presentes em todo território nacional, o que se observa, de forma geral, é o predomínio do rastreamento oportunista, sem busca ativa e convocação das mulheres na faixa etária, ações de educação em saúde e seguimento das mulheres ${ }^{46}$, o que acaba por reproduzir o padrão de maior cobertura de exames em mulheres com maior escolaridade. O Chile, por sua vez, além de não contar com um sistema universal de saúde, também não possui, ainda, um programa organizado, já que não há um sistema de convocação das mulheres da população-alvo conforme a faixa etária e periodicidade estabelecidas.

Diferente de países onde as ações de rastreamento ainda não foram implementadas, devido aos custos para infraestrutura, recursos humanos, materiais de consumo, monitoramento e vigilância, o Brasil e o Chile contam com programa de controle do câncer do colo do útero e com uma complexa rede de serviços. A redução da incidência e da mortalidade por câncer do colo do útero, de forma mais expressiva, dependerá, porém, da capacidade de ambos os países superarem os problemas e limites identificados. No Brasil, maiores esforços para garantir a qualidade dos exames, a confirmação diagnóstica e o tratamento precisam ser realizados, pois o exercício da compa- 
ração demostrou que há diferenças significativas na qualidade dos laboratórios, na abrangência do sistema de informação e na organização da rede de referência, com melhores resultados no Chile. No Brasil e no Chile, o CCU ainda é considerado um grave problema de saúde pública e as estra- tégias que buscam alterar esse cenário apontam para a estruturação do rastreamento organizado, objetivando aumentar a cobertura nas faixas etárias recomendadas e garantir o seguimento de todas as mulheres com exames alterados.

Quadro 4. Sistema de Saúde, políticas e ações de controle do câncer do colo do útero no Brasil e no Chile, 2020.

\begin{tabular}{|c|c|c|}
\hline Dimensão & Brasil & Chile \\
\hline $\begin{array}{l}\text { Contexto e } \\
\text { componentes } \\
\text { do sistema } \\
\text { público de } \\
\text { saúde }\end{array}$ & $\begin{array}{l}\text { A Constituição Federal assegura a saúde como } \\
\text { um direito de todos e dever do Estado. } \\
\text { Sistema público universal de saúde. Sistema } \\
\text { público de saúde com cobertura integral. } \\
\text { Cerca de } 24 \% \text { da população tem seguro } \\
\text { privado configurando o que se denomina } \\
\text { dupla cobertura. } \\
\text { Sem barreiras legais de acesso no sistema } \\
\text { público. } \\
\text { O Estado é o principal regulador e, além de } \\
\text { definir os macroprocessos, também estabelece } \\
\text { os mecanismos para o direcionamento da } \\
\text { assistência à saúde. Enquanto financiador } \\
\text { destina 9,47\% do Produto Interno Bruto } \\
\text { (PIB) à saúde. } \\
\text { O Ministério da Saúde tem a responsabilidade } \\
\text { de desenvolver normas e parâmetros para } \\
\text { a estruturação do SUS e aprovisionar } \\
\text { incentivos financeiros para esferas estaduais e } \\
\text { municipais. } \\
\text { No âmbito estadual, o planejamento da saúde } \\
\text { deve ser realizado de maneira regionalizada, a } \\
\text { partir das necessidades dos municípios. } \\
\text { Os municípios são responsáveis pelo } \\
\text { planejamento, organização, execução } \\
\text { e avaliação das ações de saúde locais, } \\
\text { prioritariamente, pela gestão da atenção } \\
\text { básica que é desempenhada com apoio } \\
\text { técnico e financeiro da União e dos estados. } \\
\text { As desigualdades regionais configuram } \\
\text { barreiras de acesso para parte da população } \\
\text { que enfrenta maior dificuldade na utilização } \\
\text { dos serviços de saúde. Dificuldades para } \\
\text { a fixação de especialistas e o insuficiente } \\
\text { quantitativo de profissionais em áreas } \\
\text { remotas produzem lacunas na assistência à } \\
\text { saúde. } \\
\text { A prestação de serviços públicos de saúde } \\
\text { e as ações promovidas integram uma rede } \\
\text { regionalizada e hierarquizada. Organização } \\
\text { tripartite, com direção única e autonomia em } \\
\text { cada esfera de governo }\end{array}$ & $\begin{array}{l}\text { A Constituição Nacional estabelece a } \\
\text { responsabilidade do Estado em vigiar a } \\
\text { execução das ações e serviços de saúde. } \\
\text { Sistema dual e baseado em seguros. Sistema } \\
\text { público de saúde com cobertura abrangente. } \\
\text { O sistema público (Fonasa) cobre cerca de } 76 \% \\
\text { da população e o privado (Isapres) } 14 \% \text {. } \\
\text { Contribuição obrigatória de 7\% dos } \\
\text { rendimentos para o financiamento do seguro } \\
\text { saúde. } \\
\text { O Estado é o principal regulador e além de } \\
\text { definir os macroprocessos também determina } \\
\text { os mecanismos para o direcionamento da } \\
\text { assistência à saúde. Enquanto financiador, } \\
\text { destina } 8,98 \% \text { do Produto Interno Bruto (PIB) } \\
\text { à saúde. } \\
\text { O Ministério da Saúde propõe políticas, } \\
\text { formula programas de saúde, definindo linhas } \\
\text { de ação e parâmetros para cada atividade, além } \\
\text { de assegurar o cumprimento e alocação de } \\
\text { recursos. } \\
\text { No âmbito regional, a adaptação das políticas } \\
\text { é realizada pela Secretaria Regional. Todas as } \\
\text { políticas, planos e projetos devem estar em } \\
\text { conformidade com o Plano Nacional de Saúde. } \\
\text { Os municípios são autônomos na gestão } \\
\text { e prestação dos serviços de acordo com as } \\
\text { políticas formuladas pelo Ministério da Saúde. } \\
\text { As principais barreiras de acesso estão } \\
\text { relacionadas à contribuição financeira } \\
\text { obrigatória, à centralização da assistência } \\
\text { médica na Região Metropolitana e aos custos } \\
\text { de medicamentos, consultas médicas e exames } \\
\text { que necessitam de copagamento. } \\
\text { A organização da saúde pública ocorre por } \\
\text { meio do Sistema Nacional de Serviços de } \\
\text { Saúde (SNSS). Possui uma rede de serviços } \\
\text { descentralizados em nível regional e sub- } \\
\text { regional. }\end{array}$ \\
\hline
\end{tabular}


Quadro 4. Sistema de Saúde, políticas e ações de controle do câncer do colo do útero no Brasil e no Chile, 2020.

\begin{tabular}{|c|c|c|}
\hline Dimensão & Brasil & Chile \\
\hline \begin{tabular}{|l} 
Estratégias \\
e diretrizes \\
para o \\
controle do \\
câncer do \\
colo do útero
\end{tabular} & $\begin{array}{l}\text { Triagem com citologia convencional, com } \\
\text { um exame a cada três anos, após dois exames } \\
\text { anuais negativos para mulheres de } 25 \text { e } 64 \\
\text { anos de idade. } \\
\text { Programa oportunístico, sem um sistema de } \\
\text { convite para as mulheres da população-alvo } \\
\text { participarem do rastreamento . } \\
\text { Programa de garantia da qualidade em } \\
\text { implantação. } \\
\text { Existência de um sistema de informação de } \\
\text { base populacional ainda em implementação } \\
\text { (SISCAN). } \\
\text { Dificuldades para implantação do } \\
\text { rastreamento organizado devido a } \\
\text { inexistência de um sistema de informação de } \\
\text { base populacional implantado nacionalmente. } \\
\text { Ausência de uma coordenação e } \\
\text { acompanhamento amplo e sistemático das } \\
\text { ações e de seus resultados nos diversos níveis } \\
\text { de gestão. } \\
\text { A divulgação de alguns indicadores do } \\
\text { Programa realizada pelo INCA que publica } \\
\text { periodicamente o Informativo Detecção } \\
\text { Precoce, com informações referentes aos } \\
\text { exames citopatológicos do colo do útero, } \\
\text { apresentadas por Unidade da Federação (UF) } \\
\text { e Regiões, fornecendo um panorama das } \\
\text { ações do programa. } \\
\text { A linha de cuidado determina a coleta do } \\
\text { exame na atenção primária, a confirmação } \\
\text { do exame e tratamento das lesões precursoras } \\
\text { na atenção secundária e o tratamento do } \\
\text { câncer do colo do útero na atenção terciária. } \\
\text { Dificuldades na comunicação entre os níveis } \\
\text { de atenção e os serviços assistenciais, além da } \\
\text { sub oferta dos procedimentos, especialmente } \\
\text { no nível secundário, geram interrupções e } \\
\text { atrasos no tratamento. }\end{array}$ & $\begin{array}{l}\text { Triagem com citologia convencional a cada três } \\
\text { anos para mulheres de } 25 \text { e } 64 \text { anos de idade } \\
\text { e teste de DNA-HPV com intervalos de cinco } \\
\text { anos (nas localidades onde estiver disponível) } \\
\text { para mulheres de } 30 \text { a } 64 \text { anos. } \\
\text { Programa oportunístico, sem um sistema de } \\
\text { convite para as mulheres da população-alvo } \\
\text { participarem do rastreamento. } \\
\text { Programa de garantia da qualidade realizado } \\
\text { desde } 1993 . \\
\text { Existência de um sistema de informação de } \\
\text { base populacional já implanta em todo o país } \\
\text { (SIGGES). } \\
\text { Possibilidade de transição para o rastreamento } \\
\text { organizado favorecido pela existência de um } \\
\text { sistema de informação de base populacional } \\
\text { e obrigatoriedade de inscrição na APS para } \\
\text { acessar o sistema público de saúde. } \\
\text { Forte controle ministerial sobre as metas } \\
\text { estabelecidas determina a realização da busca } \\
\text { ativa e o seguimento para toda mulher com } \\
\text { exame alterado. } \\
\text { Divulgação dos resultados do Programa } \\
\text { realizada por meio de um relatório anual de } \\
\text { avaliação que analisa o grau de resolutividade } \\
\text { em relação à detecção, diagnóstico, } \\
\text { estadiamento e tratamento, identificando } \\
\text { as lacunas existentes no cumprimento de } \\
\text { garantias explícitas, constituindo uma guia } \\
\text { para tomada de decisão e alocação de recursos. } \\
\text { A linha de cuidado determina a coleta do } \\
\text { exame na atenção primária, a confirmação } \\
\text { do exame e tratamento das lesões precursoras } \\
\text { na atenção secundária e o tratamento do } \\
\text { câncer do colo do útero na atenção terciária. O } \\
\text { monitoramento por parte das garantias Auge } \\
\text { estabelece o prazo para cada ação ser realizada. }\end{array}$ \\
\hline
\end{tabular}

Fonte: Elaboração própria com base na bibliografia pesquisada.

\section{Colaboradores}

IB Claro e LD Lima contribuiu na concepção, delineamento, análise e interpretação dos dados, na redação do artigo e sua revisão crítica e na aprovação da versão a ser publicada. PF Almeida contribuiu na redação do artigo e sua revisão crítica e na aprovação da versão a ser publicada.

\section{Agradecimentos}

LD Lima e PF Almeida são Bolsistas de Produtividade em Pesquisa do Conselho Nacional de Desenvolvimento Científico e Tecnológico (CNPq). LD Lima conta também com o apoio da Fundação de Amparo à Pesquisa do Estado do Rio de Janeiro (FAPERJ) na realização de estudos sobre a política de saúde no Brasil e no estado do Rio de Janeiro. 


\section{Referências}

1. Arby M, We-iderpass E, Sanjosé S, Bruni L, Saraiya M, Ferlay J, Bray F. Estimates of incidence and mortality of cervical cancer in 2018: a worldwideanalysis. Lancet Glob Health 2020; 8: e191-e203. Published Online, 2019.

2. Hull R, Mbele M, Makhafola T, Hicks C, Wang SM, Reis RM, Mehrotra R, Mkhize-Kwitshana Z, Kibiki G, Bates DO, Dlamini Z. Cervical cancer in low and middle-income countries (Review). OncolLetters 2020; 20:2058-2074.

3. Goss PE, Lee BL, Badovinac-Crnjevic T, Strasser-Weippl K, Chavarri-Guerra Y, St Louis J, Villarreal-Garza C, Unger-Saldaña K, Ferreyra M, Debiasi M, Liedke PE, Touya D, Werutsky G, Higgins M, Fan L, Vasconcelos C, Cazap E, Vallejos C, Mohar A, Knaul F, Arreola H, Batura R, Luciani S, Sullivan R, Finkelstein D, Simon S, Barrios C, Kightlinger R, Gelrud A, Bychkovsky V, Lopes G, Stefani S, Blaya M, Souza FH, Santos FS, Kaemmerer A, Azambuja E, Zorilla AF, Murillo R, Jeronimo J, Tsu V, Carvalho A, Gil CF, Sternberg C, Dueñas-Gonzalez A, Sgroi D, Cuello M, Fresco R, Reis RM, Masera G, Gabús R, Ribeiro R, Knust R, Ismael G, Rosenblatt E, Roth B, Villa L, Solares AL, Leon MX, Torres-Vigil I, Covarrubias-Gomez A, Hernández A, Bertolino M, Schwartsmann G, Santillana S, Esteva F, Fein L, Mano M, Gomez H, Hurlbert M, Durstine A, Azenha G. Planning cancer control in Latin America and the Caribbean. Lancet Oncol 2013; 14(5):391-436.

4. Luciani S, Andrus JK. A Pan American Health Organization strategy for cervical cancer prevention and control in Latin America and the Caribbean - Reprod. Health Matters 2008; 59:59-66.

5. Raúl M, Robles C. Research needs for implementing cancer prevention and early detection in developing countries: from scientists to implementers perspectives. BioMed Res Intern 2019; (n. esp):9607803.

6. Organización Panamericana de La Salud (OPAS). Situación de los Programas para la Prevención y el Control del Cáncer Cervicouterino: evaluación rápida mediante encuesta en 12 países de América Latina. Washington, D.C.: OPS; 2010.

7. Brawley OW. Cancer screening: a general perspective In Cancer prevention and screening - Concepts, principles and controversies. Edited by Rosalind A. Eeles, Christine D. Berg, Jeffrey S. Tobias. John Wiley \& Sons: Oxford; 2019.

8. World Health Organization (WHO). Cancer Control: knowledge into action: WHO guide for effective programmes, Module 2. Prevention. Geneva: WHO; 2007.

9. Zeferino LC. O desafio de reduzir a mortalidade por câncer do colo do útero. Rev Bras Ginecol Obstet 2008; 30(5):213-215.

10. Capote Negrin LG. "Epidemiologyof Cervical Cancer in LatinAmerica." Ecancer medical science 2015; 9: 577. PMC.
11. Lemp JM, De Neve JW, Bussmann H, Chen S, Manne-Goehler J, Theilmann M, Marcus ME, Ebert C, Probst C, Tsabedze-Sibanyoni L, Sturua L, Kibachio JM, Moghaddam SS, Martins JS, Houinato D, Houehanou C, Gurung MS, Gathecha G, Farzadfar F, Dryden-Peterson S, Davies JI, Atun R, Vollmer S, Bärnighausen T, Geldsetzer P. Lifetime Prevalence of Cervical Cancer Screening in 55 Low- and Middle-Income Countries. JAMA 2020; 20; 324(15):1532-1542.

12. Chile. Ministerio de Salud. Guía Clínica AUGE. Cáncer Cervicouterino ( $\mathrm{CaCu}$ ). Santiago: Minsal; 2015.

13. Brasil. Ministério da Saúde. Instituto Nacional de Câncer José Alencar Gomes da Silva (INCA). Diretrizes Brasileiras para o Rastreamento do Câncer do Colo do Útero. Coordenação de Prevenção e Vigilância. 2a ed. Rio de Janeiro: INCA; 2016.

14. Marmor T, Freeman R, Okma K. Comparative Perspectives and Policy Learning in the World of Health Care. J Comp Policy Analysis: Res Practice 2005; 7(4): 331-348.

15. Rose R. Learning from comparative public policy. New York, NY: Routledge; 2005.

16. Conill EM, Fausto MCR, Giovanella L. Contribuições da análise comparada para um marco abrangente na avaliação de sistemas orientados pela atenção primária na América Latina. Rev Brasil Saude Materno Infantil 2010; 10(Supl.1):S151-S27.

17. Teixeira LA, Fonseca CMO, coordenadores. De Doença desconhecida a problema de saúde pública: o INCA e o controle do Câncer no Brasil. Rio de Janeiro: Ministério da Saúde; 2007.

18. Instituto Nacional de Câncer José Alencar Gomes da Silva (INCA). Viva Mulher 20 anos: história e memória do controle do câncer do colo do útero e de mama no Brasil: catálogo de documentos. Rio de Janeiro: INCA; 2018.

19. Lanza SS, Sepúlveda CV, Olate MB, Espejo CC. Aplicación de metodología de marco lógico para el análisis del Programa Nacional de Pesquisa y Control del Cáncer Cervicouterino en Chile. Rev Chil Obstet Ginecol 2010; 75(5):294-299.

20. Cordeiro GV, Pérez SC, Iñarrea AF, Simón DV, Reboredo CR, Couceiro EN, Ramón y Cajal C. ¿Por qué no se vacunan nuestras pacientes?: Motivos por los que las pacientes fuera de los programas de vacunación sistemática, con infección por el virus del Papiloma Humano, deciden decir no a la vacuna. Rev Chil Obstet Ginecol 2014; 79(5):390-395.

21. Zanini NV, Prado BS, Hendges RC, Santos CA, Callegari FVR, Bernuci MP. Motivos para recusa da vacina contra o Papilomavírus Humano entre adolescentes de 11 a 14 anos no município de Maringá-PR. Rev Brasil Med Fam Comum 2017; 12(39):1-13.

22. Organização para a Cooperação e Desenvolvimento Económico (OCDE). Relatórios Econômicos OCDE: Brasil. Paris: OECD; 2018.

23. Organización de Cooperación y Desarrollo Económicos (OCDE). Estudios económicos de la OCDE: Chile. Paris: OCDE; 2018. 
24. Parikh S, Brennan P, Boffetta P. Meta-analysis of social inequality and the risk of cervical cancer. Int J Cancer 2003; 105:687-691.

25. Nuche-Berenguer B, Sakellariou D. Socioeconomic determinants of cancer screening utilisation in Latin America: A systematic review. PloS ONE 2019; 14(11): e0225667.

26. Wunsch Filho V, Antunes JLF, Boing AF, Lorenzi RL. Perspectivas da investigação sobre determinantes sociais em câncer. Physis 2008; 18(3):427-450.

27. Chile. Ministerio de Salud (MS); Subsecretaría de Salud Pública. Decreto com Fuerza de Ley 1. Santiago: MS; 2005.

28. Chile. Ministerio de Salud (MS). Subsecretaría de Salud. Pública. AUGE 85. Garantías de Oportunidad. Santiago: MS; 2019.

29. Instituto Nacional de Câncer (INCA). Plano de ação para redução da incidência e mortalidade por câncer do colo do útero: sumário executivo. Rio de Janeiro: INCA; 2010.

30. Urrutia MT. Cáncer Cervico uterino en Chile: análisis de um nuevo paradigma preventivo. Temas de la agenda pública 2015; 10(78)

31. Vidal C, Hoffmeister L, Biagini L. Tendencia de la mortalidad por cáncer de cuello uterino en Chile: aplicación de modelos de regresión joinpoint. $\mathrm{Rev} \mathrm{Pa}$ nam Salud Publica 2013; 33(6):407-413.

32. International Agency for Research on Cancer (IARC). Handbooks of Cancer Prevention. Vol. 10. Cervix Cancer Screening. Lyon: IARCPress; 2005.

33. Organização Pan-Americana da Saúde (OPAS). Controle integral do câncer do colo do útero. Guia de práticas essenciais. Washington, DC: OPAS; 2016.

34. Ribeiro, CM, Azevedo e Silva, G. Avaliação da produção de procedimentos da linha de cuidado do câncer do colo do útero no Sistema Único de Saúde do Brasil em 2015. Epidemiol Serv Saude 2018; 27(1):e20172124.

35. Farias ACB, Barbieri AR. Seguimento do câncer de colo de útero: Estudo da continuidade da assistência à paciente em uma região de saúde. Esc Anna Nery 2016; 20(4).

36. Araujo JR MLC, Santana DA, Almeida LB, Quintana SBS, Silva GRF, Fonseca RCSP. Quality in cytopathology: ananalysis of the internal quality monitoring indicators of the Instituto Nacional de Câncer. J Bras Patol Med Lab 2015; 51(2):102-107.

37. Instituto Nacional de Câncer (INCA). Coordenação de Prevenção e Vigilância. Divisão de Detecção Precoce e Apoio à Organização de Rede. Manual de gestão da qualidade para laboratório de citopatologia. $2^{\mathrm{a}}$ ed. rev. ampl. Rio de Janeiro: INCA; 2016.

38. Tomasi E, Oliveira TF, Fernandes PAA, Thumé, E, Silveira DS, Siqueira FV, Duro SMS, Saes MO, Nunes BP, Fassa, AG, Facchini LA. Estrutura e processo de trabalho na prevenção do câncer de colo de útero na Atenção Básica à Saúde no Brasil: Programa de Melhoria do Acesso e da Qualidade - PMAQ. Rev Bras Saude Matern Infant 2015; 15(2):171-180.
39. Fernandes NFS, Galvão JR, Assis MMA, Almeida PF, Santos AM. Acesso ao exame citológico do colo do útero em região de saúde: mulheres invisíveis e corpos vulneráveis. Cad Saude Publica 2019; 35(10).

40. Almeida PF, Oliveira SC, Giovanella L. Integração de rede e coordenação do cuidado: o caso do sistema de saúde do Chile. Cien Saude Colet 2018; 23(7).

41. Gallardo K, Varas L, Gallardo M. Inequality of opportunity in health: evidence from Chile. Rev Saude Publica 2017; 4(51):110.

42. Chile. Ministerio de Salud (MS). Subsecretaría de Salud. Pública Decreto N. 42.449. Aprueba garantías explícitas em salud del régimen general de Garantías em Salud. Santiago: MS; 2019.

43. Wiesner-Ceballos C, Murillo Moreno RH, Piñeros Petersen M, Tovar-Murillo SL, Cendales Duarte R, Gutiérrez MC. Control del câncer cervicouterino en Colombia: la perspectiva de los actores del Sistema de salud. Rev Panam Salud Publica 2009; 25(1):1-8.

44. Sarfati D. Why social inequalities matter in the cancer continuum In Reducing social inequalities in cancer: evidence and priorities for research / edited by S. Vaccarella, J. Lortet-Tieulent, R. Saracci, D.I. Conway, K Straif, CP Wild. IARC Library Cataloguing in Publication Data. Geneva: World Health Organization; 2019.

45. Menvielle G, Kulhánová I, Mackenbach JP. Assessing the impact of a public health intervention to reduce social inequalities in cancer In: Reducing social inequalities in cancer: evidence and priorities for research /edited. Vaccarella S, Lortet-Tieulent J, Saracci R, Conway DI, Straif K, Wild CP. IARC Library Cataloguing in Publication Data, World Health Organization; 2019.

46. Galvão JR, Almeida PF, Santos AM, Bousquat A. Percursos e obstáculos na Rede de Atenção à Saúde: trajetórias assistenciais de mulheres em região de saúde do Nordeste brasileiro. Cad Saude Publica 2019; 35(12):e00004119.

Artigo apresentado em 20/11/2020

Aprovado em 25/05/2021

Versão final apresentada em 27/05/2021

Editores-chefes: Romeu Gomes, Antônio Augusto Moura da Silva 
